\title{
Peculiar property of noble gases and its explanation through the Enskog-Vlasov model
}

\author{
E. S. Benilor and M. S. Benilor
}

(Dated: July 13, 2021)

\begin{abstract}
A new observation is presented that the ratio of the critical and triple-points temperatures, $T_{c r} / T_{t p}$, of neon, argon, krypton, and xenon fit within a narrow interval: $T_{c r} / T_{t p}=1.803 \pm 0.5 \%$, and the same applies to the density ratio: $n_{c r} / n_{t p}=0.3782 \pm 1.7 \%$ (of the two remaining noble gases, helium does not have a triple point and, for radon, $n_{t p}$ is unknown). We explain this peculiar property by the fact that the molecules of noble gases are nearly spherical, as a result of which they satisfy the Enskog-Vlasov (EV) kinetic model based on the approximation of hard spheres. The EV model has also allowed us to identify two more parameter combinations which are virtually the same for all noble gases.
\end{abstract}

\section{INTRODUCTION}

Noble gases have been studied for decades - and yet noone has observed that their parameters form certain nondimensional 'invariants', which hardly change from gas to gas. In the present paper, we identify four such invariants and explain their universality using the Enskog-Vlasov (EV) model.

The EV kinetic equation comprises the Enskog collision integral for dense fluids [1] and a Vlasov term describing the van-der-Waals force (similar to that for the electromagnetic force in plasma 2 2). The first version of the EV model [3, 4] was based on the original form of the Enskog integral - which, as shown in Ref. [5], does not comply with the Onsager relations. Ref. 5 has also proposed a modification of the Enskog integral that is free from this shortcoming, which was incorporated in the EV model in Refs. 6, 7]. A restriction of the coefficients of the Enskog-Vlasov equation guaranteeing that it satisfies an H-theorem has been formulated in Refs. [8 10, and Ref. 11] proposed a version of the EV equation that does satisfy this restriction and conserves energy as well (all of the previous versions did not).

The EV model-The Enskog integral is based on an approximation of the molecules by hard spheres - hence, the Enskog-Vlasov model should be best applicable to noble fluids, whose molecules are nearly spherical (e.g., they have neither dipole nor quadrupole asymmetry). It has been tested for neon, argon, krypton, and xenon [11, and it was shown that the thermodynamic properties of these fluids are indeed consistent with the constraints implied by the EV model. In particular, the per-molecule internal energy $U_{p m}$ and entropy $S_{p m}$ are reasonably ac-

\footnotetext{
* Department of Mathematics and Statistics, University of Limerick, V94 T9PX, Ireland; Eugene.Benilov@ul.ie http://www.staff.ul.ie/eugenebenilov/hpage/

† Departamento de Física, CCCEE, Universidade da Madeira, Largo do Município, 9000 Funchal, Portugal; Instituto de Plasmas e Fusão Nuclear, Instituto Superior Técnico, Universidade de Lisboa, Portugal; benilov@uma.pt http://fisica.uma.pt/ingles/pessoal/Mikhail’Benilov/
}

curately described by

$$
\begin{gathered}
U_{p m}=\frac{3}{2} k_{B} T-\frac{1}{2} E n, \\
S_{p m}=k_{B}\left[\ln \frac{T^{3 / 2}}{n}-\Theta\left(D^{3} n\right)\right],
\end{gathered}
$$

where $k_{B}$ is the Boltzmann constant, $T$ is the temperature, $n$ is the number density, $D$ is the effective molecular diameter, the Vlasov parameter $E$ characterizes the strength of the van-der-Waals force. The function $\Theta(\xi)$ describes the non-ideal part of the fluid's entropy and, thus, vanishes at zero density,

$$
\Theta(0)=0,
$$

whereas the EV model implies [11] that

$$
\Theta^{\prime}(0)=\frac{2 \pi}{3},
$$

where $\Theta^{\prime}(\xi)=\mathrm{d} Q / \mathrm{d} \xi$. As shown in Ref. [11, Eqs. (1)(2) correspond to the following equation of state $(\mathrm{EoS})$ :

$$
p=n k_{B} T\left[1+D^{3} n \Theta^{\prime}\left(D^{3} n\right)\right]-\frac{1}{2} E n^{2},
$$

where $p$ is the pressure, and the following expression for the per-molecule Gibbs free energy:

$$
\begin{aligned}
G_{p m}=k_{B} T[ & \ln \left(n T^{-3 / 2}\right) \\
& \left.+\Theta\left(D^{3} n\right)+D^{3} n \Theta^{\prime}\left(D^{3} n\right)\right]-E n,
\end{aligned}
$$

Before using the EV model, one should calibrate it, i.e. fix $E, D$, and $\Theta(\xi)$. Note that the first two parameters are specific to the fluid, whereas $\Theta(\xi)$ is supposed to be a universal function characterizing all noble fluids.

The Vlasov parameter $E$ was determined for each of the four fluids under consideration by fitting a linear dependence to the empiric data [12] for $U-\frac{3}{2} k_{B} T$ on the critical isobar, as a function of $n$ (see Table I) [13. Table I also presents the parameters of the critical and triple points of the four fluids [12] to be used later. 


\begin{tabular}{|c|c|c|c|c|c|c|c|}
\hline & $E N_{A}^{2}\left(\mathrm{~J} \mathrm{l} / \mathrm{mol}^{2}\right)$ & $n_{t p} / N_{A}(\mathrm{~mol} / \mathrm{l})$ & $T_{t p}(\mathrm{~K})$ & $p_{t p}(\mathrm{bar})$ & $n_{c r} / N_{A}(\mathrm{~mol} / \mathrm{l})$ & $T_{c r}(\mathrm{~K})$ & $p_{c r}(\mathrm{bar})$ \\
\hline $\mathrm{Ne}$ & 51.6 & 62.059 & 24.562 & 0.43464 & 23.882 & 44.4918 & 26.786 \\
\hline Ar & 325 & 35.465 & 83.8058 & 0.68891 & 13.4074 & 150.687 & 48.630 \\
\hline $\mathrm{Kr}$ & 550 & 29.197 & 115.77 & 0.73503 & 10.85 & 209.48 & 55.250 \\
\hline $\mathrm{Xe}$ & 983 & 22.592 & 161.4 & 0.81748 & 8.4 & 289.733 & 58.420 \\
\hline
\end{tabular}

TABLE I. The dimensional parameters of neon, argon, krypton, and xenon. The Vlasov parameter $E$, $n_{t p}$, and $n_{c r}$ are normalized using the Avogadro constant $N_{A}$.

As shown in Ref. 11, the effective molecular diameter $D$ can be related to the triple-point density $n_{t p}$,

$$
D=n_{t p}^{-1 / 3}
$$

whereas the function $\Theta(\xi)$ will be discussed later.

\section{THE MAIN RESULT}

Introduce the following nondimensional parameters:

$$
\begin{gathered}
\alpha=\frac{T_{c r}}{T_{t p}}, \quad \beta=\frac{n_{c r}}{n_{t p}}, \\
\gamma=\frac{k_{B} T_{t p}}{E n_{t p}}, \quad \delta=\frac{p_{c r}}{k_{B} T_{c r} n_{c r}} .
\end{gathered}
$$

If one of these parameters is calculated using the data from Table 1 for the four noble fluids under consideration, the resulting four values (see Table II) fit into a fairly narrow interval:

$$
\begin{array}{ll}
\alpha=1.803 \pm 0.5 \%, & \beta=0.3782 \pm 1.7 \%, \\
\gamma=0.06186 \pm 3.1 \%, & \delta=0.2959 \pm 2.4 \% .
\end{array}
$$

If neon is excluded, the universal nature of invariants (8) - 9ecomes a little more evident:

$$
\begin{array}{ll}
\alpha=1.802 \pm 0.4 \%, & \beta=0.3748 \pm 0.9 \%, \\
\gamma=0.06010 \pm 0.4 \%, & \delta=0.2905 \pm 0.6 \% .
\end{array}
$$

The fact that neon is slightly off can be explained by the fact that its triple-point temperature is considerably lower than those of the other four fluids; as a result, its liquid phase may be influenced by quantum effects. Note that the van-der-Waals EoS yields for $\delta$ a universal value of 0.375 , which however differs significantly from that observed for noble gases.

To explain the constancy of invariants (8)-(9), assume that the four fluids under consideration are described by

\begin{tabular}{|l||c|c|c|c|}
\hline \hline & $\frac{T_{c r}}{T_{t p}}$ & $\frac{n_{c r}}{n_{t p}}$ & $\frac{k_{B} T_{t p}}{E n_{t p}}$ & $\frac{p_{c r}}{k_{B} T_{c r} n_{c r}}$ \\
\hline \hline $\mathrm{Ne}$ & 1.8114 & 0.38483 & 0.063774 & 0.30320 \\
\hline $\mathrm{Ar}$ & 1.7980 & 0.37805 & 0.060454 & 0.28950 \\
\hline $\mathrm{Kr}$ & 1.8094 & 0.37161 & 0.059942 & 0.29237 \\
\hline $\mathrm{Xe}$ & 1.7951 & 0.37181 & 0.060427 & 0.28870 \\
\hline \hline
\end{tabular}

TABLE II. The nondimensional parameters of neon, argon, krypton, and xenon.

the EV model with the same function $\Theta(\xi)$. Recall also that a fluid's critical and triple points both lie on the curve in the $(n, T)$ plane, representing the relationship between the parameters of the saturated vapor and liquid. This curve can be obtained through the Maxwell construction, i.e. by equating the two phases' pressures and chemical potentials (for a single-component fluid, the latter coincides with the per-molecule Gibbs free energy). Recalling thus (5)-(6) and introducing the nondimensional variables

$$
\xi=D^{3} n, \quad \tau=\frac{k_{B} D^{3}}{E} T,
$$

we obtain

$$
\begin{gathered}
\xi_{v} \tau\left[1+\xi_{v} \Theta^{\prime}\left(\xi_{v}\right)\right]-\frac{\xi_{v}^{2}}{2}=\xi_{l} \tau\left[1+\xi_{l} \Theta^{\prime}\left(\xi_{l}\right)\right]-\frac{\xi_{l}^{2}}{2} \\
\tau\left[\ln \xi_{v}+\Theta\left(\xi_{v}\right)+\xi_{v} \Theta^{\prime}\left(\xi_{v}\right)\right]-\xi_{v} \\
=\tau\left[\ln \xi_{l}+\Theta\left(\xi_{l}\right)+\xi_{l} \Theta^{\prime}\left(\xi_{l}\right)\right]-\xi_{l}
\end{gathered}
$$

where the subscripts $v$ and $l$ mark the parameters of the vapor and liquid phases, respectively.

Physically, vapor and liquid can coexist only if $T<$ $T_{c r}$ - hence, Eqs. 111 - 12 have a non-trivial solution $\left(\xi_{v} \neq \xi_{l}\right)$ only if $\tau<\tau_{c r}$, where $\tau_{c r}$ is the nondimensional $T_{c r}$. Straightforward calculations show that $\tau_{c r}$ and the 
corresponding nondimensional density $\xi_{c r}$ are determined by

$$
\begin{gathered}
3 \xi_{c r}^{2} \Theta^{\prime \prime}\left(\xi_{c r}\right)+\xi_{c r}^{3} \Theta^{\prime \prime \prime}\left(\xi_{c r}\right)=1, \\
\tau_{c r}=\frac{\xi_{c r}}{1+2 \xi_{c r} \Theta^{\prime}\left(\xi_{c r}\right)+\xi_{c r}^{2} \Theta^{\prime \prime}\left(\xi_{c r}\right)} .
\end{gathered}
$$

To find the parameters of the triple point, observe that calibration (7) and nondimensionalization (10) imply

$$
\left(\xi_{l}\right)_{t p}=1
$$

Thus, the nondimensional triple-point temperature can be found by letting in Eqs. $111-12 \xi_{l}=1$ and $\tau=\tau_{t p}$,

$$
\begin{gathered}
\xi_{v} \tau_{t p}\left[1+\xi_{v} \Theta^{\prime}\left(\xi_{v}\right)\right]-\frac{\xi_{v}^{2}}{2}=\tau_{t p}\left[1+\Theta^{\prime}(1)\right]-\frac{1}{2} \\
\tau_{t p}\left[\ln \xi_{v}+\Theta\left(\xi_{v}\right)+\xi_{v} \Theta^{\prime}\left(\xi_{v}\right)\right]-\xi_{v} \\
=\tau_{t p}\left[\Theta(1)+\Theta^{\prime}(1)\right]-1 .
\end{gathered}
$$

Eq. 16 - 17 determine $\tau_{t p}$ and the corresponding nondimensional density $\xi_{v}$ of the saturated vapor (in what follows, the latter will not be needed).

Recalling nondimensionalization (10), relationship (7), the latter's nondimensional equivalent (15), and EoS (5), we can express invariants (8) - 9 in the form

$$
\begin{gathered}
\alpha=\frac{\tau_{c r}}{\tau_{t p}}, \quad \beta=\xi_{c r}, \\
\gamma=\tau_{t p}, \quad \delta=1+\Theta^{\prime}(1)-\frac{1}{2 \tau_{t p}} .
\end{gathered}
$$

Now, the universality of these parameters follows from the mere fact that Eqs. (13)- 14 and $(16)-(17)$ - which determine $\xi_{c r}, \tau_{c r}$, and $\tau_{t p}$ - do not include any fluidspecific parameters.

Note that none of our conclusions derived so far depends on the specific form of the function $\Theta(\xi)$, as long as it is the same for all four fluids under consideration.

\section{CALIBRATING THE EV MODEL}

The invariants found turn out to be helpful for the inner workings of the EV model, as they help us to finish calibrating it for noble fluids [by fixing $\Theta(\xi)$ ].

We shall approximate $\Theta(\xi)$ by a fifth-degree polynomial. Given restrictions (3)-(4), this amounts to

$$
\Theta=\frac{2 \pi}{3} \xi+a_{2} \xi^{2}+a_{3} \xi^{3}+a_{4} \xi^{4}+a_{5} \xi^{5}
$$

We have deduced the coefficients $a_{2,3,4,5}$ from the requirement that the invariants $\alpha, \beta, \gamma$, and $\delta$ assume the correct
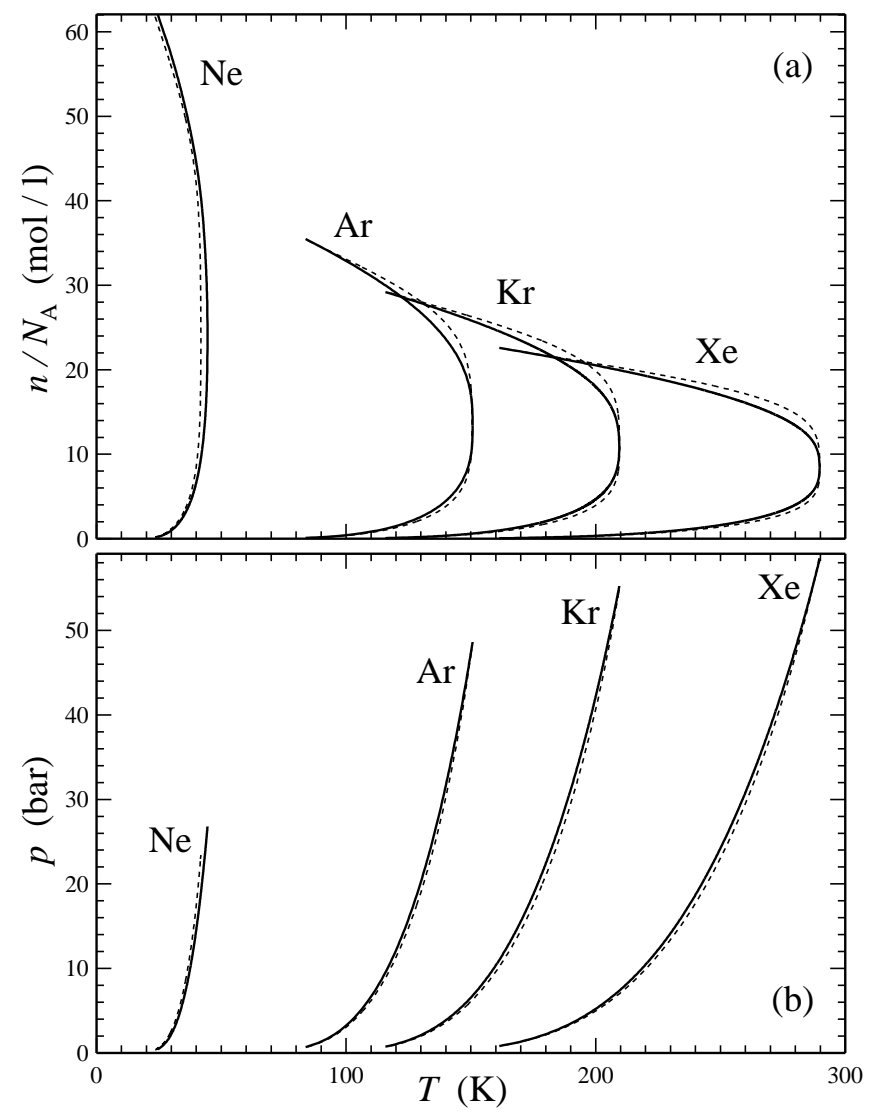

FIG. 1. The parameters of phase transitions of noble fluids. The solid curves show the empiric data [12] and the dotted ones, the results obtained through the EV model. (a) The molar densities of the saturated vapor and liquid (the upper and lower parts of the curves, respectively) vs. $T$. (b) The pressure of the saturated vapor vs. $T$.

values, where the "correct" means "the average over argon, krypton, and xenon" (to eliminate quantum effects - no matter how weak they are - neon was excluded). To do so, we used Eqs. (13)-(14), (16)-(17), and $\sqrt{18}-(20)$ to relate $a_{2,3,4,5}$ to $\alpha, \beta, \gamma$, and $\delta$ - and thus obtained

$$
\begin{array}{ll}
a_{2}=-1.8103, & a_{3}=9.6325, \\
a_{4}=-12.831, & a_{5}=6.2501 .
\end{array}
$$

Since the function $\Theta(\xi)$ is now known, we can calculate the parameters of the saturated vapor and liquid from Eqs. (11) - 12, and use Eq. (5) to find the EoS. These results have been compared to the corresponding empiric data [12].

Since we have calibrated the EV model using the parameters of the critical and triple points of argon, krypton, and xenon, it comes as no surprise that their phasetransition properties are described well - and even those of neon are described reasonably accurately (see Fig. 1). More surprisingly, the same calibration also provides an accurate description of the noble fluids' EoS (see Fig. 2) 14 . 

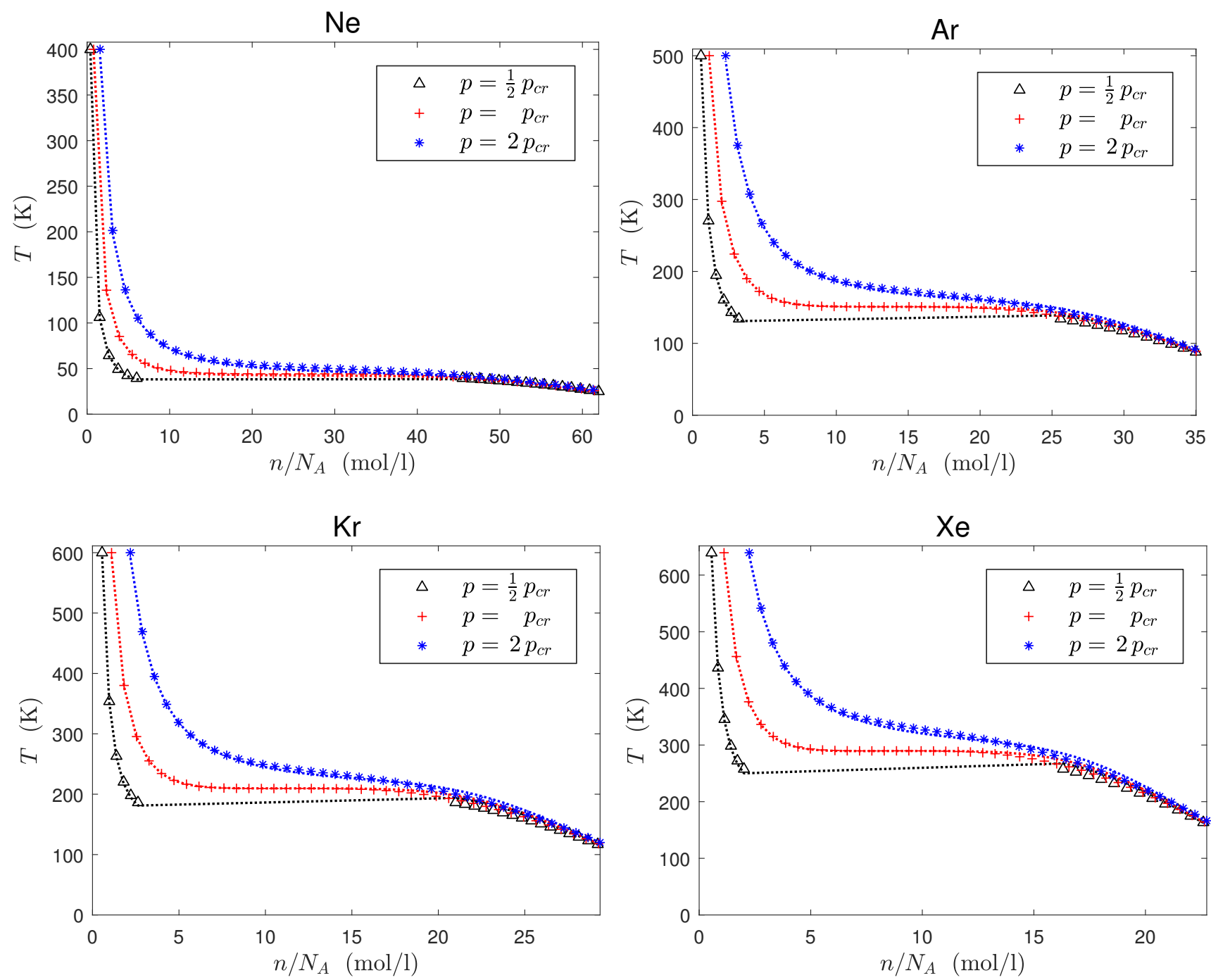

FIG. 2. A comparison of the Enskog-Vlasov EoS (5), (7), 20)- 22) (dotted curves) and the empiric data 12] (non-connected symbols) for neon, argon, krypton, and xenon. The panel labels (circled) indicate the corresponding fluid.

\section{SUMMARY AND CONCLUDING REMARKS}

The main result of the present work is an observation that certain characteristics of phase transitions of noble fluids hardly change from fluid to fluid. Other groups of substances do not seem to have this peculiar property, not even the halogens (which are the closest neighbors of noble gases in the periodic table). Indeed, for fluorine, chlorine, bromine, and iodine, $T_{c r} / T_{t p}=2.409 \pm 12.1 \%$, i.e. the spread of this parameter is considerably larger than that for noble fluids. We attribute this difference to the fact that the halogen molecules are strongly asymmetric, so the Enskog-Vlasov model does not describe them as accurately as it does noble fluids.

Note that the constancy of invariants (8) - 9 can also be explained using basic dimensional analysis and the assumption that, in the fluid(s) under consideration, the potential of the van-der-Waals force involves no more than 2 dimensional parameters - such as, for example, the Lennard-Jones potential truncated at the molecule's diameter. This approach was used in Ref. [? ] to explain constancy of the (critical-point) parameter $\delta$, but it has never been applied to triple-point characteristics, such as $\alpha, \beta$, and $\gamma$. Furthermore, the EV model allows one to explain the constancy of all four characteristics regardless of the number of parameters in the van-der-Waals force.

Finally, we emphasize that we do not advertise the EV model as a means of predicting properties of fluids, as there are tools (e.g., Refs. 12, 15]) which do this job with a higher accuracy. It should rather be used as a kinetic equation, and the accuracy of its thermodynamic predictions is just an indicator of its overall accuracy. This is an important point, as several version of the EnskogVlasov kinetic equation have been used for applications 
(see Refs. [16, 17] and references therein).

\section{ACKNOWLEDGMENTS}

We acknowledge the support of the Science Foundation Ireland through grant 12/IA/1683, and that of Fundação para a Ciência e a Tecnologia of Portugal through project Pest-OE/UID/FIS/50010/2013.
[1] D. Enskog, Kungl. Svenska Vetenskaps Akad. Handl. 63, 1 (1922).

[2] A. A. Vlasov, Sov. Phys. Usp. 10, 721 (1968).

[3] L. de Sobrino, Can. J. Phys. 45, 363 (1967)

[4] M. Grmela, J. Stat. Phys. 3, 347 (1971)

[5] H. van Beijeren and M. H. Ernst, Physica 68, 437 (1973)

[6] J. Karkheck and G. Stell, J. Chem. Phys. 75, 1475 (1981)

[7] G. Stell, J. Karkheck, and H. van Beijeren, J. Chem. Phys. 79, 3166 (1983)

[8] M. Grmela and L. S. Garcia-Colin, Phys. Rev. A 22, 1295 (1980).

[9] M. Grmela and L. S. Garcia-Colin, Phys. Rev. A 22, 1305 (1980).

[10] M. Grmela, Can. J. Phys. 59, 698 (1981).

[11] E. S. Benilov and M. S. Benilov, Phys. Rev. E 97, 062115 (2018)

[12] P. J. Linstrom and W. G. Mallard, "NIST chemistry web-
We are also grateful to V. Yu. Zitserman for helpful remarks. book, NIST standard reference database number 69," (1997).

[13] In Ref. [11, $E$ was calculated using the isobar with the pressure being twice the critical pressure. The difference between that result and the present one is less than $0.5 \%$, which proves the robust nature of the employed method for determining $E$.

[14] Note that the EoS-derived calibration proposed in Ref. [1] is sufficiently accurate only for the EoS, but is less so for the parameters of the saturated vapor and liquid (which we did not realize at the time).

[15] C. Tegeler, R. Span, and W. Wagner, J. Phys. Chem. Ref. Data 28, 779 (1999)

[16] A. Frezzotti and P. Barbante, Mech. Eng. Rev. 4, 16 (2017).

[17] A. Frezzotti, L. Gibelli, D. A. Lockerby, and J. E. Sprittles, Phys. Rev. Fluids 3, 054001 (2018). 